\title{
AICAPA - Um Ambiente Inteligente e Colaborativo para Apoio à Produção Acadêmica
}

\author{
Crediné Silva Menezes ${ }^{1}$ \\ Karla Samantha Bezerra Vale ${ }^{1}$ \\ Patrícia de Oliveira Cruz ${ }^{1}$
}

\begin{abstract}
${ }^{1}$ Departamento de Informática - Universidade Federal do Espírito Santo (UFES) Av. Fernando Ferrari, 514, Campus de Goiabeiras - 29.075-910 - Vitória - ES - Brasil \{credine, karlasamantha, pattrycie\}@gmail.com
\end{abstract}

\begin{abstract}
Resumo. Considerada a maior e a principal fonte de informações no mundo, a Web está se tornando cada vez mais importante para o pesquisador, pois contém uma grande variedade de artigos acadêmicos que podem contribuir para a elaboração de suas pesquisas. No entanto, nesse mar de informação, torna-se difícil encontrar o que é relevante para o usuário. No presente trabalho apresentamos a proposta de um ambiente colaborativo na web, que tem por objetivo apoiar a revisão da literatura, atividade fundamental para dar suporte a todo trabalho acadêmico, através de técnicas de Recomendação e de Recuperação da Informação baseada em conhecimentos sobre o contexto do problema de pesquisa.
\end{abstract}

Palavras-chave: ambientes colaborativos, revisão da literatura, técnicas de recomendação, técnicas de recuperação da informação.

\begin{abstract}
Considered the largest and the main source of information in the world, the Web is becoming increasingly important for the researched, it contains a wide variety of academic articles that can contribute to the development of their research. However, in this sea information, it becomes difficult to find what is relevant to the user. In this paper we present a proposal for a collaborative web environment, which aims to support the literature review, key activity to support all academic work, through technical recommendation and information retrieval based on knowledge about the context of research problem.
\end{abstract}

Keywords: collaborative environments, recommendation techniques, literature review, information retrieval techniques.

\section{Introdução}

O crescimento exponencial na quantidade de informações na web tem tornado a busca de documentos uma tarefa problemática. Segundo Delicato et. al. (2001), esse grande aumento das informações disponibilizadas pela Internet, embora favoreça a disseminação do conhecimento e a obtenção de produtos e serviços, também torna a procura de material relevante um verdadeiro desafio.

O grande volume e diversidade de conteúdo disponível na web têm trazido dificuldades na busca e seleção de material interessante. De acordo com ISC Internet Domain Survey (Birukov et.al, 2005), em julho de 2004 existiam 285.139.107 hosts na Internet e este número cresceu 22\% desde janeiro de 2004. De acordo com a Internet World Stats (Primo et. al., 2010) dos anos 2000 até 2009 a taxa de crescimento de informações foi de $380 \%$ e o número de domínios alcançou segundo a Internet Systems Consortium a quantia de 730 milhões em janeiro de 2010. Pelas estatísticas devemos ter um volume e uma variedade muito grande de trabalhos publicados, o que ocasiona a sobrecarga 
Pesquisadores encontram muitas dificuldades em realizar o levantamento da literatura para seu trabalho, pois existe a dificuldade de encontrar documentos relevantes ou não saber formular consultas na web. Diante do grande volume de informação armazenado e distribuído entre diversos websites direcionados à pesquisa acadêmica (ex.: Portal de Periódicos, IEEExplore), o trabalho de busca e seleção dos candidatos mais relevantes pode ser maçante e demorado, o que delonga a redação de um trabalho acadêmico, em qualquer nível (TCC, dissertação, tese etc). Tal situação sugere estudos do uso de mecanismos que facilitem a localização de documentos relevantes de forma a lidar com a grande massa de conteúdo disponível na web. Assim, a utilização de uma ferramenta que automatize o processo de busca e seleção de materiais relevantes para o usuário, facilitaria a fase de revisão da literatura que, como se sabe, consome grande parte do tempo despendido em um trabalho acadêmico.

Em um ambiente colaborativo, diversas pessoas podem se comunicar e trocar ideias e, assim, é construído o conhecimento. Nessa interação, pessoas podem se comunicar e esclarecer dúvidas sobre sua pesquisa acadêmica, porém nem todos terão tempo para escutar e responder as perguntas, ou sequer buscar em artigos e documentos a resposta para seu questionamento. Dessa forma, busca-se com este projeto oferecer a comunidade acadêmica um ambiente colaborativo que auxilie estudantes e pesquisadores no enriquecimento de suas atividades acadêmicas através do uso de técnicas de recomendação, recuperação de artigos e de esclarecimento de dúvidas.

Assim, este trabalho está dividido em seis seções. A seção 2 mostra os estudos relacionados e apresenta uma breve comparação entre eles e a proposta deste artigo. A seção 3 traz a fundamentação teórica que se baseia nosso trabalho. A seção 4 apresenta a proposta e a arquitetura do ambiente proposto. Por fim, na seção 5 apresentamos as considerações finais.

\section{Trabalhos Relacionados}

Dentro da pesquisa acadêmica existem algumas ferramentas que fornecem ambientes que apoiam esta tarefa, contudo esses ambientes ainda apresentam algumas deficiências, tais como ausência de modelos para fichamentos, acompanhamento colaborativo, recomendação direta etc.

Para a análise destas ferramentas de apoio dentre as opções disponíveis, foram selecionadas as seguintes ferramentas: Mendeley, EndNote, PaperBox, Zotero e StArt, que serão brevemente descritas nesta seção.

Uma das ferramentas de maior destaque nesta área é o Mendeley ${ }^{1}$, desenvolvida por Glyph \& Cog, é um gerenciador de referências bibliográficas além de uma rede social acadêmica. Algumas das características mais marcantes são a possibilidade de criar grupos de discussão e com isso potencializar a possibilidade de colaboração com diversos outros pesquisadores interessados em problemas correlatos. A ferramenta também dispõe de um sistema de busca, que permite localizar artigos, pessoas e grupos, além de disponibilizar acesso aos documentos.

O EndNote ${ }^{2}$ é uma ferramenta software online para gerenciamento de referências, utilizado para gerenciar bibliografias e referências ao escrever ensaios e artigos, sendo desenvolvida pela Thomson Reuters. Com este software, o usuário pode criar um banco de dados personalizado de referências, organizar e armazenar as citações em bases de dados chamados bibliotecas. É um formatador de citação, a ferramenta cria, automaticamente, referências, notas de rodapé e bibliografias nos seus documentos

\footnotetext{
${ }^{1} \mathrm{http}: / /$ mendeley.com

$2 \mathrm{http}: / /$ endnote.com

3 http://paper-box.co
} 
escritos e possui uma interface de busca, permitindo que o usuário pesquise em catálogos on-line e em bancos de dados diretamente do Endnote.

PaperBox ${ }^{3}$ é uma ferramenta baseada em nuvem que permite a organização e o acesso das referências bibliográficas de qualquer sistema operacional. Assim como algumas das ferramentas aqui citadas, o PaperBox permite a colaboração conjunta de artigos, além do compartilhamento entre usuários. Ainda conta com um plugin do Microsoft Word, que permite que o usuário faça citações de acordo com o contexto, sem se preocupar com a formatação.

Zotero $^{4}$ é uma ferramenta gratuita, desenvolvida por Roy Rosenzwieg Center for History and New Media, que permite organizar, colecionar, citar e compartilhar referências bibliográficas. Algumas das características de destaque deste software são a de reconhecimento do conteúdo do navegador do usuário e permite que este o adicione na biblioteca da ferramenta; a indexação automática dos arquivos do texto, que facilita a busca dos documentos relevantes. Além de funcionalidades como o compartilhamento (privado ou público) e sincronização entre as diferentes plataformas.

StArt $^{5}$ é uma ferramenta de apoio a Revisões Sistemáticas desenvolvida pelo Laboratório de Pesquisa em Engenharia de Software da UFSCar. Seu objetivo é dar suporte ao Planejamento, Execução e Análise Final de uma Revisão Sistemática, independentemente do assunto ou área de pesquisa, tornando-a mais ágil, precisa e replicável. StArt fornece suporte as atividades do processo de revisão sistemática da literatura, exceto à pesquisa automatizada de estudos primários em banco de dados eletrônicos.

Os critérios de comparação entre as ferramentas escolhidas e a ferramenta proposta estão dispostos de forma resumida na Tabela 01, que é apresentada a seguir.

Tabela 01 - Tabela Comparativa

\begin{tabular}{|l|c|c|c|c|c|}
\hline \multicolumn{1}{|c|}{ Coftware } & Mendeley & EndNote & PaperBox & Zotero & StArt \\
\hline Versão para a web? & Sim & Sim & Sim & Sim & Não \\
\hline Custo & Gratuito & $\$ 250$ & Gratuito & Gratuito & Gratuito \\
\hline Realiza recomendações? & Não & Não & Não & Não & Não \\
\hline $\begin{array}{l}\text { Busca e recuperação } \\
\text { automática de } \\
\text { informações relevantes? }\end{array}$ & Não & Não & Não & Não & Não \\
\hline Soluciona dúvidas? & Não & Não & Não & Não & Não \\
\hline $\begin{array}{l}\text { Apoio à Revisão da } \\
\text { Literatura? }\end{array}$ & Não & Não & Não & Não & Sim \\
\hline $\begin{array}{l}\text { Armazenamento em } \\
\text { nuvem? }\end{array}$ & Sim & Sim & Sim & Sim & Não \\
\hline
\end{tabular}

\footnotetext{
${ }^{4}$ http://lapes.dc.ufscar.br/tools/start_tool

${ }^{5}$ http://www.zotero.org/
} 
Observando as características e deficiências de cada ferramenta aqui descritas, podemos destacar que o diferencial do trabalho proposto são as funcionalidades de busca, seleção e recomendação personalizada de artigos relevantes para o desenvolvimento do projeto de pesquisa dos usuários do ambiente, além da recomendação de grupos e pessoas para incentivar a colaboração e facilitar a realização do trabalho acadêmico.

Sobre o critério de busca e recuperação de informação, o sistema pesquisará artigos, pessoas etc de forma automática, selecionando os relevantes ao usuário, de acordo com seu perfil de interesses. Além disso, o módulo de fichamento, onde os usuários colocarão as ideias principais e farão anotações e destaques nos artigos lidos, conterá um sistema de esclarecimento de dúvidas capaz de extrair trechos de texto que possam solucionar questionamentos dos acadêmicos. A intenção do ambiente é auxiliar os usuários na revisão da literatura, uma vez que, é uma tarefa exaustiva e demorada devido ao esforço consumido na busca e seleção de artigos relevantes na web.

Dessa forma, as ferramentas apresentadas não fornecem apoio adequado a estudantes e pesquisadores no processo de revisão da literatura. Enquanto, a ferramenta aqui proposta oferece meios eficientes para uma revisão mais completa.

\section{Fundamentação Teórico-Metodológica}

A solução que apresentaremos para este problema está apoiada em duas áreas da Inteligência Artificial, a recomendação de artigos e sistemas de esclarecimento de dúvidas, sendo estas as duas macrofuncionalidades do ambiente, que serão descritas nas subseções seguintes.

\subsection{Sistemas de Recomendação}

De acordo com Reategui e Cazella (2005), sistemas de recomendação podem ser definidos como sistemas que procuram auxiliar indivíduos a identificarem conteúdos de interesse em um conjunto de opções que poderiam caracterizar uma sobrecarga. Eles empregam técnicas de filtragem de informação e de aprendizagem de máquina para gerar recomendações apropriadas aos usuários.

Segundo Balabanovic e Shoham (1997), sistemas de recomendação são classificados em categorias, baseados em como as recomendações são feitas:

- Baseada em conteúdo: tenta-se recomendar itens semelhantes àqueles que um determinado usuário tenha gostado;

- Colaborativa: identifica os usuários cujos gostos são semelhantes aos do usuário dado e recomenda itens que eles tenham gostado;

- Híbrida com marcador: Envolve combinar a abordagem baseada em conteúdo e colaborativa.

Existem deficiências nas abordagens baseada em conteúdo e colaborativa e, por isso, surgiu a abordagem híbrida com marcador, que é o foco desse trabalho. A abordagem baseada em conteúdo apresenta limitações como, por exemplo, o uso de sinônimos dificulta o entendimento do conteúdo dos textos, a desestruturação dos dados (como, sons, vídeos e figuras) é difícil de ser analisada e a super especialização do sistema em tópicos frequentes no perfil do usuário, pois ao aprender sobre o perfil do usuário, o sistema não inova em suas recomendações. Segundo Reategui e Cazella (2005), a coleta de informações dos usuários em filtragem colaborativa apresenta alguns problemas:

1. Problema do primeiro avaliador: quando um novo item aparece no banco de dados não existe maneira deste ser recomendado para o usuário até que mais informações sejam obtidas através de outros usuários; 
2. Problema de pontuação esparsas: o objetivo dos sistemas de filtragem colaborativa é ajudar pessoas, focando em documentos lidos ou itens adquiridos. Caso o número de usuários seja pequeno em relação ao volume de informações no sistema existe um grande risco das pontuações tornarem-se muito esparsas;

3. Similaridade: caso um usuário tenha gostos que variam do normal, este terá dificuldades para encontrar outros usuários com gostos similares, sendo assim suas recomendações podem se tornar pobres.

No ambiente aqui proposto, o sistema de recomendação recomendará artigos, pessoas e grupos relevantes aos usuários a partir do perfil do mesmo que conterá seus interesses, podendo ser obtidos de forma implícita através da observação do seu comportamento ou de forma explícita, através de formulários que serão preenchidos no momento da realização do cadastro no ambiente.

O perfil de interesses será representado como uma ontologia, com o intuito de obter um perfil preciso para que os resultados da recomendação sejam adequados aos interesses do usuário. Uma estratégia de recomendação utilizada será a avaliação dos usuários, ou seja, após ler um artigo recomendado o usuário poderá avaliá-lo para que outras pessoas acreditem na qualidade do mesmo. No entanto, para que isso possa funcionar corretamente, o sistema apresentará uma relação de perguntas (até cinco) para que o usuário possa avaliar o artigo em todos os seus aspectos e o sistema fará uma média ponderada das respostas para obter a avaliação daquele trabalho. Além disso, a técnica utilizada para gerar as recomendações será a abordagem híbrida, na qual é utilizada a técnica de filtragem baseada em conteúdo e a colaborativa para que as limitações de cada uma sejam resolvidas, pois uma tende a completar a outra. Caso haja alguma limitação que não possa ser resolvida com as duas abordagens, outras técnicas poderão ser utilizadas para resolver tais problemas.

\subsection{Sistemas de Esclarecimento de Dúvidas}

Sistemas de Esclarecimento de Dúvidas, ou Question and Answering Systems (QAS), procuram ocupar a lacuna de resultados personalizados para o usuário, que é deixada pelos sistemas de busca em larga escala. O objetivo dos QAS é extrair, automaticamente, respostas corretas e relevantes ao usuário, fazendo uso de técnicas de extração/recuperação de informação e processamento de linguagem natural (Gupta e Gupta, 2012).

Este tipo de sistema possui, em sua maioria, uma arquitetura baseada em quatro (04) módulos: base de dados, módulo de análise do questionamento, módulo para busca e extração da resposta e um módulo para gerar e apresentar a resposta selecionada para o usuário (Amorim et. al., 2011).

Grande parte dos sistemas QA são desenvolvidos com o intuito de participar da TREC (Text REtrieval Conference), que reúne um grande número de publicações acerca das evoluções desses sistemas. Dentro do campo educacional, exploram-se sistemas de esclarecimento de dúvidas como meio de interação entre o aluno e um especialista ou a interação entre o aluno e o ambiente computacional, com o intuito de sanar questionamentos do aluno ou até gerados pelo próprio especialista, esta forma de interação é de fundamental importância tanto para a consolidação do conhecimento, quanto a sua validação.

Neste trabalho, o papel desse tipo de sistema é auxiliar o usuário em seu fichamento, em especial a seção de dúvidas. O ambiente faz um comparativo entre as palavras-chave dos questionamentos ou dúvidas inseridas pelo acadêmico e as palavras encontradas nos artigos presentes na base de dados. A partir deste comparativo, serão extraídos trechos de texto que possam sanar os questionamentos prévios. 


\subsection{Ambientes Colaborativos e Revisão da Literatura}

Neste trabalho adotamos ambientes colaborativos para que usuários interajam com outras pessoas para facilitar na elaboração de trabalhos acadêmicos e focamos em dar suporte à revisão da literatura, que é imprescindível na elaboração de qualquer projeto acadêmico.

Ambientes Colaborativos são tipos de software que apoiam e promovem o trabalho em grupo apoiados por computadores. Sob essa ótica, estes ambientes abrem uma nova perspectiva para colaboração em grupo, possibilitando que seus participantes interajam através de simulações do mundo real ou imaginário (Filippo et. al., 2007). Segundo Brito et. al. (2004), a importância dos ambientes colaborativos é notada no mundo globalizado, desde prover o compartilhamento de informações locais até a permitir a interação entre pessoas distantes.

Atualmente, existe uma enorme necessidade de realizar atividades e solucionar problemas de modo colaborativo. De acordo com Brito et.al. (2004), no desenvolvimento de projetos ou na aprendizagem colaborativa por meio de problemas, esta característica de interação entre membros de um ambiente possui importância primordial, uma vez que habilidades individuais precisam ser combinadas com as de outras pessoas de forma a produzirem um trabalho de qualidade. Para Haguenauer et. al. (2007), a proposta central da criação de um Ambiente Colaborativo consiste em proporcionar um espaço de construção coletiva do conhecimento, onde cada participante é autor e colaborador e pode usufruir o resultado do esforço de todos.

A revisão da literatura, também conhecida como "revisão bibliográfica", "estado da arte" ou "estado do conhecimento", corresponde a uma das etapas da pesquisa científica visando, de acordo com Santos (2012), a demonstrar o estágio atual da contribuição acadêmica em torno de um determinado assunto. É a fase vital para elaboração de qualquer trabalho acadêmico, uma vez que, os pesquisadores precisam investigar em que ponto se encontra o estado do conhecimento sobre aquele assunto. Por isso, é uma atividade exaustiva que envolve buscar, selecionar e analisar trabalhos já publicados sobre o tema em diversas fontes de informação que cresce exponencialmente a cada ano.

De acordo com Moreira (2004), muitas vezes consome-se mais tempo tentando identificar se determinado estudo já foi realizado anteriormente do que o realizando de fato. Já Bento (2012) afirma que embora apresente vários benefícios, a revisão de literatura é muitas vezes o trabalho mais enfadonho para alguns estudiosos, pois corresponde proporcionalmente à maior parte do processo de pesquisa. Deve-se, a isso, ao fato do pesquisador ter que ler diversos trabalhos sobre o tema para que ele possa ter condições de formular um problema de pesquisa interessante, pertinente e original.

\section{Uma Proposta de Ambiente}

Com base nos aspectos que identificamos, como sendo pontos importantes na realização de uma revisão da literatura assim, como nos pontos que ainda não encontram respostas satisfatórias nos sistemas disponíveis, elaboramos a proposta de um sistema inteligente colaborativo. Sob essa perspectiva, o ambiente proposto neste trabalho tem como objetivo principal fornecer aos pesquisadores e estudantes, uma ferramenta que automatize o processo de busca e seleção de artigos relevantes na web para reduzir os esforços realizados no processo de revisão da literatura. Além disso, a ferramenta conta com apoio de diversas outras funcionalidades que contribuirão significativamente no tempo gasto utilizado na elaboração de trabalhos acadêmicos. 
Esta seção apresenta a proposta do ambiente usando técnicas de recomendação e esclarecimento de dúvidas, abordando a sua descrição e o detalhamento de sua arquitetura.

\subsection{Funcionamento do Ambiente}

A interação do usuário começa com o seu cadastramento, onde são fornecidos dados pessoais, profissionais, acadêmicos, login e senha. Nesta fase, é construído seu perfil inicial de interesses, para que o sistema possa recomendar grupos, pessoas e artigos com os dados do seu perfil. Assim, será possível que o usuário encontre pessoas e grupos com interesses correlatos.

A ferramenta permitirá que usuários compartilhem e colaborem com diversas pessoas, com a possibilidade de formação de grupos: públicos e privados, através destes o usuário pode compartilhar documentos instantaneamente. Os membros do grupo podem visualizar documentos e pastas que cada usuário adicionar ao grupo, permitindo manter-se atualizado com os seus colaboradores. Assim, é possível comentar e iniciar a discussão quando quiser e assistir o progresso de projetos ao longo do tempo.

O sistema recomendará grupos, usuários, com interesses em comum, além de artigos aos usuários do ambiente acadêmico. Assim, o usuário terá a possibilidade de ter contato com pessoas e grupos com afinidade, auxiliando-o na elaboração de projetos de pesquisa. A recomendação também pode ser feita diretamente de usuário para usuário.

O sistema permitirá ao usuário avaliar os artigos, à medida que ele for fazendo os fichamentos, permitindo que o sistema recomende os que tiverem avaliação positiva, seguindo critérios definidos pelo sistema. Dessa forma, a recomendação se tornará mais confiável, pois pessoas irão ler o artigo que outras leram e afirmaram ser bom através de tal avaliação.

Para que haja recomendação será preciso que cada usuário e grupo tenham seus perfis de interesse. O perfil do usuário será atualizado continuamente pelo sistema que estará monitorando qualquer ação dele no ambiente. O perfil do grupo será definido pelo usuário que criou o grupo. O perfil do usuário poderá ser editado e visualizado pelo usuário, o que aumenta a precisão das recomendações.

O usuário poderá criar projetos individuais ou em grupo através de convites para outras pessoas que vão participar do projeto. Projetos serão separados em: andamento ou concluído. Artigos e outros documentos poderão ser compartilhados, para que todos os participantes do projeto possam trabalhar em conjunto. No projeto, usuários poderão usar um editor de texto colaborativo, no qual a equipe poderá escrever sobre o trabalho ao mesmo tempo. Qualquer alteração que ocorrer no texto, cada usuário participante do projeto recebe uma notificação sobre a alteração. Assim, toda a equipe acompanhará o andamento do projeto.

O ambiente trará ao usuário a possibilidade de criar, editar e deletar fichamentos que foram elaborados a partir dos artigos lidos e, após a leitura, o usuário poderá recomendar o artigo a outros usuários, como comentado anteriormente. Durante a leitura dos artigos, que será feito no próprio sistema, o usuário poderá introduzir notas e destacar partes do texto, fazendo uma pequena resenha do mesmo. Dessa forma, o sistema faz uma análise de tais anotações e, baseadas nelas, a ferramenta cria um perfil de interesses para que ela possa recomendar outros artigos, pessoas e grupos com base em perfis comuns.

Nesses fichamentos, o usuário tem a opção de alterar os níveis de acesso e edição aos demais participantes, sendo elas: editar, visualizar, comentar etc. Ainda neste módulo, o ambiente fornece uma estrutura para listagem e esclarecimento de dúvidas. Esta funcionalidade tem por principal objetivo sanar questionamentos do usuário em 
relação à leitura de um artigo, este relata suas indagações na seção de dúvidas do módulo e o ambiente traça um paralelo entre as palavras-chave contidas neste relato e as palavras encontradas em artigos relacionados ao tema explorado. Em seguida, são extraídos trechos dos textos que possam solucionar a questão ou auxiliar o usuário na busca para a resposta mais adequada.

Outra característica importante sobre o sistema diz respeito à listagem dos artigos recomendados e recuperados para o usuário, esta funcionalidade apresentará uma descrição de cada artigo, contendo informações como: título, abstract/resumo, autores, local e ano de publicação. Esses dados serão recuperados a partir das referências bibliográficas dos artigos relevantes ao tema, com isso, o usuário poupará tempo no detalhamento dos artigos em sua listagem. Além disso, o sistema exibirá o abstract/resumo de cada referência dos artigos lidos e fará uma análise das palavras do mesmo, comparando-as com as do artigo em leitura e calculando um índice de importância para que sejam feitas mais recomendações ou novas buscas e recuperações de publicações. A seguir é apresentada a arquitetura do sistema.

\subsection{Arquitetura do Sistema}

Nesta seção é apresentada a arquitetura do sistema proposto, incluindo as relações entre os módulos e agentes que a compõem, representada pela Figura1. O ambiente é composto por cinco agentes que interagem entre si para realizar as suas tarefas. Os agentes que compõe o sistema são:

- Agente de Interface: exibe o conteúdo ao usuário, ou seja, toda e qualquer informação que o Agente Recomendador encaminhe ao usuário é repassado para este agente. Além disso, este agente coleta toda a interação do usuário no ambiente que considere relevante e encaminha ao Agente de Usuário para que a base de perfis seja atualizada;

- Agente de Usuário: recebe os dados do usuário quando o Agente de Interface recebe uma informação relevante sobre o usuário. O agente atualiza a Base de Dados do Usuário quando o Agente de Interface informa dados relevantes e, em seguida, emite os dados do usuário ao Agente Recomendador para que seja encaminhado ao Agente de Busca e Recuperação, a fim de buscar artigos na web e armazená-los na base de dados local. Além disso, é responsável por garantir que o conjunto de perfis reflita os interesses dos usuários adaptando-se a eles ao longo do tempo;

- Agente de Busca e Recuperação: agente responsável por invocar mecanismos de busca e recuperação de artigos existentes na web, obter seus resultados e armazená-los numa base de dados local. Além disso, o agente realiza buscas dentro do ambiente, podendo está relacionadas a artigos, pessoas ou grupos; também acumula a função de recuperação de informação destas mesmas categorias previamente citadas;

- Agente de Recomendação: é responsável por sugerir documentos, pessoas e grupos semelhantes aos perfis dos usuários. O agente recebe os dados alterados do perfil do usuário pelo Agente de Usuário, realiza cálculos de similaridade e solicita ao Agente de Busca e Recuperação para localizar documentos, pessoas e grupos adequados àquele usuário. O Agente de Busca e Recuperação encaminha essas informações ao Agente Recomendador e o mesmo encaminha ao Agente de Interface para que seja exibido ao usuário.

- Agente de Dúvidas: este agente é responsável por identificar as dúvidas levantadas pelo usuário, comparar as palavras-chaves contidas nestes questionamentos e palavras-chave em artigos relacionados ao tema explorado; 
também responsável pela extração de trechos de texto de artigos da base de dados a partir das palavras-chaves comparadas; este agente também encaminha dúvidas que não obtiveram resposta através de trechos de texto para usuários especialistas na área, reconhecidos através do Agente do Usuário.

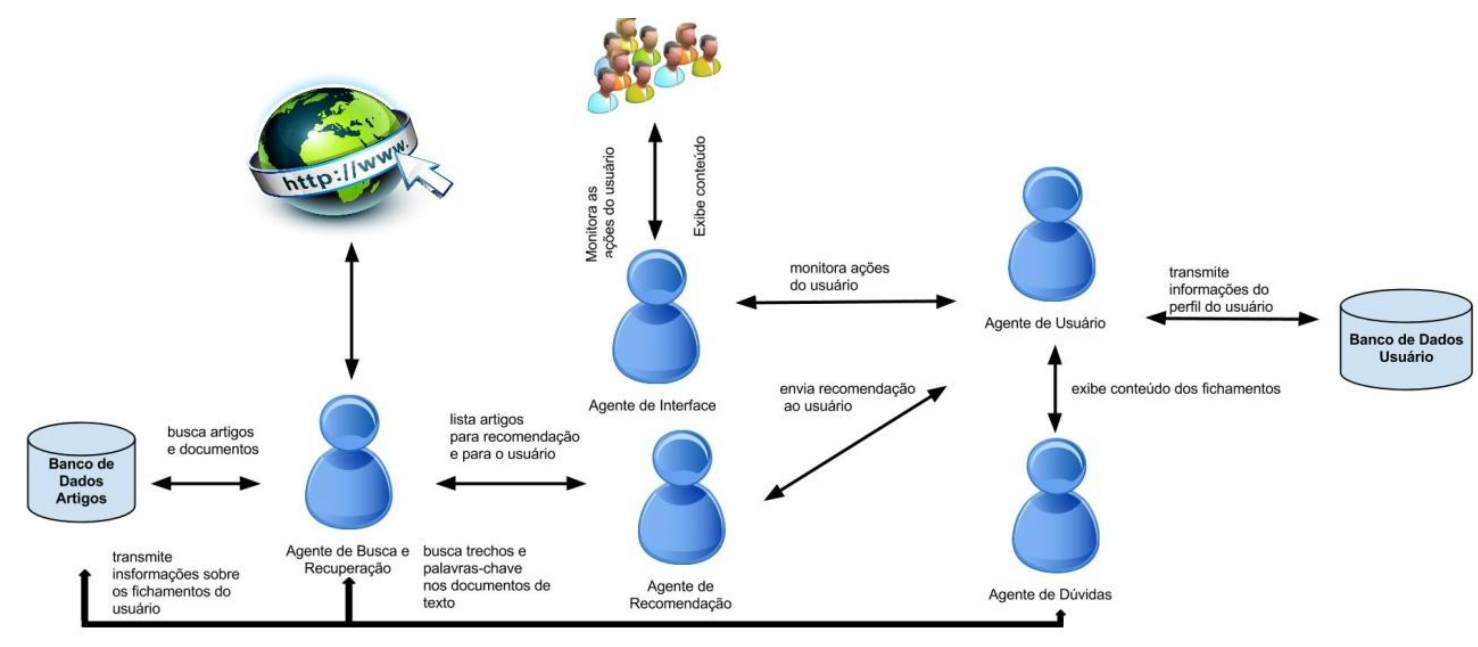

Figura 1 - Arquitetura do Sistema Proposto

\subsection{Ambiente de Desenvolvimento}

O ambiente colaborativo será desenvolvido para a web, utilizando a linguagem de programação $\mathrm{PHP5}^{6}$, com auxílio da ferramenta NetBeans ${ }^{7}$, o servidor será o Apache e o sistema gerenciador de banco de dados, $\mathrm{MySQL}^{8}$. Para a implementação das ontologias será utilizada a ferramenta Protegé ${ }^{9}$. Com essa ferramenta é possível implementar ontologias em diversos formatos como, por exemplo, OWL (Ontology Web Language) que é uma linguagem para representar ontologias na web e que está sendo cada vez mais utilizada em um número maior de aplicações. A ferramenta se constitui, também, em um espaço de armazenamento de textos na web (armazenamento em nuvem).

\section{Considerações Finais}

Neste trabalho apresentamos a proposta de um ambiente colaborativo e personalizado que utiliza técnicas de recomendação e de esclarecimento de dúvidas para que possa contribuir com estudantes e pesquisadores na elaboração de trabalhos acadêmicos. Foi proposta uma solução para resolver o problema que acompanha estudantes e pesquisadores durante a fase de revisão da literatura.

A recomendação de pessoas e grupos poderá aumentar a interação entre os usuários do ambiente, permitindo o aumento do nível de conhecimento $\mathrm{e}$ enriquecimento do projeto de pesquisa. A recomendação de artigos ocorrerá de acordo com o perfil do usuário que será alimentado durante o cadastramento no sistema e durante as ações do usuário no ambiente. A utilização de um sistema de esclarecimento de dúvidas permitirá que o ambiente encontre respostas aos questionamentos surgidos durante a realização dos fichamentos dos artigos.

\footnotetext{
${ }^{6}$ http://php.net

${ }^{7}$ https://netbeans.org/

${ }^{8}$ http://www.mysql.com/

${ }^{9} \mathrm{http}: / /$ protege.stanford.edu/
} 
Como trabalhos futuros serão realizados, além da implementação da proposta descrita, testes do modelo em um cenário real com o intuito de avaliar o impacto de uma ferramenta deste gênero no meio acadêmico. Este trabalho está sendo desenvolvido como projeto de pesquisa acadêmica realizada no Laboratório de Informática na Educação (LIED), da Universidade Federal do Espírito Santo.

\section{Referências}

AMORIM, M. T. C. F.; CURY, D.; MENEZES, C. S.; Um sistema inteligente baseado em ontologia para apoio ao esclarecimento de dúvidas. Em: Anais do XXII SBIE - XVII WIE, 2011.

BALABANOVIC, M.; SHOSAM, Y. Fab: Content-based, collaborative recommendation. Em: Communications of the ACM, 40(3):66-72, 1997.

BENTO, A. Como fazer uma revisão da literatura: Considerações teóricas e práticas. Em: Revista JA (Associação Acadêmica da Universidade da Madeira), nº 65, ano VII (pp. 42-44). ISSN: 1647-897, 2012.

BIRUKOV, A.; BLANZIERI, E.; GIORGINI, P. Implicit: An Agent Based Recommendation System for Web Search. Em: AAMAS'05 (July 25-29,20 05, Utrecht, Netherlands), 2005.

BRITO, R. F.; PEREIRA, A. T. C. Um estudo para ambientes colaborativos e suas ferramentas. Em: Congresso Nacional de Ambientes Hipermídia para Aprendizagem. UFSC, Florianópolis, 2004.

DELICATO, F. C. et al. Multiagentes para a Filtragem de Páginas Web. Em: Simpósio Brasileiro de Redes de Computadores, SBRC, 19, 2001. Florianópolis: UFSC, 2001.

FILIPPO, D.; RAPOSO, A.; ENDLER, M.; FUKS, H. Ambientes Colaborativos de Realidade Virtual e Aumentada. Em: Realidade Virtual e Aumentada - Conceitos, Projeto e Aplicações, Cap. 9, pp 168-191, Porto Alegre, 2007.

GUPTA, P.; GUPTA, V.; A survey of text question answering techniques. Em: International Journal of Computer Applications (0975 - 8887), 2012.

HAGUENAUER, C.; KOPKE, R. C. M.; VICTORINO, A. L. Q.; FILHO, F. C. Ambientes Colaborativos de Aprendizagem no apoio ao ensino presencial: a experiência do programa de pós-graduação em educação da UFRJ, Em: Colabor@, RICESU, Vol. 4, No. 16, novembro, 2007.

MORAIS, E. A. M.; AMBRÓSIO, A. P. I. Ontologias: conceitos, usos, tipos, metodologias, ferramentas e linguagens. UFG, Goiás, GO, 2007.

MOREIRA, W. Revisão de literatura e desenvolvimento científico: conceitos e estratégias para confecção. Janus, Lorena, v. 1, n. 1, p. 19-30, jul./dez. 2004.

PRIMO, T.T.; VICARI, R. M.; SILVA, J. M. C. Rumo ao Uso de Metadados Educacionais em Sistemas de Recomendação. Em: Simpósio Brasileiro de Informática na Educação - SBIE 2010, 2010, João Pessoa. XXI Simpósio Brasileiro de Informática na Educação, 2010

REATEGUI, E.; CAZELLA, S. C. Sistemas de Recomendação. Em: XXV Congresso da Sociedade Brasileira de Computação. Unisinos. São Leopoldo, RS, 2005.

SANTOS, V. O que é e como fazer Revisão da Literatura na Pesquisa Teológica. Em: Fides reformata, 17.1 (2012): 89-104. 